\title{
Research and Design of Car Active Reversing Safety Control System SUN Yang ${ }^{1}$, LV Liya ${ }^{2, a}$, ZHU ShuLin ${ }^{3}$, DAI Ting ${ }^{4}$, ZHAO Fei ${ }^{5}$, SONG Jie ${ }^{6}$ 1,2,3,4,5,6 College of Automobile and Traffic Engineering, Nanjing Forestry University, Nanjing 210037, China \\ avliya@163.com
}

Keywords: Keywords: E-hydraulic power steering system; Reversing trajectory; Mathematical model; Distance sensor; Steering wheel angle sensor

\begin{abstract}
When the vehicle reversing of the visual blind spot or may have obstacles, prone to traffic accidents such as collision. This article uses the distance sensor and steering angle sensor for real-time monitoring of the vehicle when reversing, based on establishing vehicle reversing trajectory mathematical model, to assist characteristic, system of electronically controlled hydraulic power steering and braking system of active mirror active adjustment, to avoid traffic accidents when reversing the purpose. The system also adopted to control the speed of the vehicle, equipped with reversing safety alarm device, take the initiative to give prompt risk, reduce the probability of occurrence of reversing collision accident in a certain extent.
\end{abstract}

\section{Introduction}

In recent years, with economic development and car ownership increased, there was a great increase in the number of traffic accidents when reversing. Because car driver has blind spots when reversing, the driver has no clear judgment of obstacles behind the vehicle, operational errors and other reasons, there are often traffic accidents. Therefore, it is necessary to develop a proactive accident prevention reversing car control system.

The automotive active safety reversing control system in this paper is a reversing system that actively assist the driver in parking so as to increase the safety. Its main role is to use distance measuring sensor mounted on the rear bumper to measure the actual distance between the obstacle and the position of the body in reverse and at the same time, combine with the car's body parameters to calculate the trajectory model car reversing when judging the degree of risk when reversing according to the value of the steering wheel angle sensor; in addition, through the vehicle control bus system to control boost the degree of electronically controlled hydraulic power systems, engine fuel injection and ignition parameters so as to control object trajectory and speed. Finally, to provide information and warnings by reversing alarm and reminder system for the driver to be informed of the situation around the vehicle barrier, thus improving driving safety.

\section{Working principle and its mathematical model of the system}

To facilitate the study, the control system classified the vehicle reversing the situation into two modes: linear reversing and steering servo reversing type. When reversing, the control system determine the angle of rotation reversing mode through the steering wheel angle sensor: If the angle is greater than $\alpha$ ( $\alpha$ is a specify value about $5^{\circ}$ and growing), the system determines the reversing is accompanied by steering; if the angle is less than $\alpha$, the system determines the linear reversing car.

Active reversing system is shown in Figure 1. [1] When the car started reversing, the system will measure a steering angle $\alpha$ ( $\alpha$ value decided by the driver habits), according to the steering angle and vehicle parameters, you can create car parking geometric model as shown in figure 4, we can predict cars running track. At the same time, while the ultrasonic sensor measures the distance to the obstacle cars, which determine whether this angle reversing is safe, if the security is reduced steering, activation of the alarm to alert the driver to reduce the steering angle $\alpha$, if necessary stop automatic braking steering control; if the case of security, it is determined whether the reverse meet the requirements, and if not on the direction to increase the angle $\alpha$, the current angle can be 
maintained to meet the continued backing.

Reversing system of Linear reverse mode works as in figure 2. When the car started reversing, the driver turns on the active side mirror system switch while turning the steering wheel is detected by the MCU, if turn left, then control rear mirror in the left side; if turn right, then control the rear mirror on the right. [2]MCU control rear view mirror to set the angle, namely rear wheel contact with the ground position, by the end to be reversing, MCU control rear mirror back positive, then switch off the system.

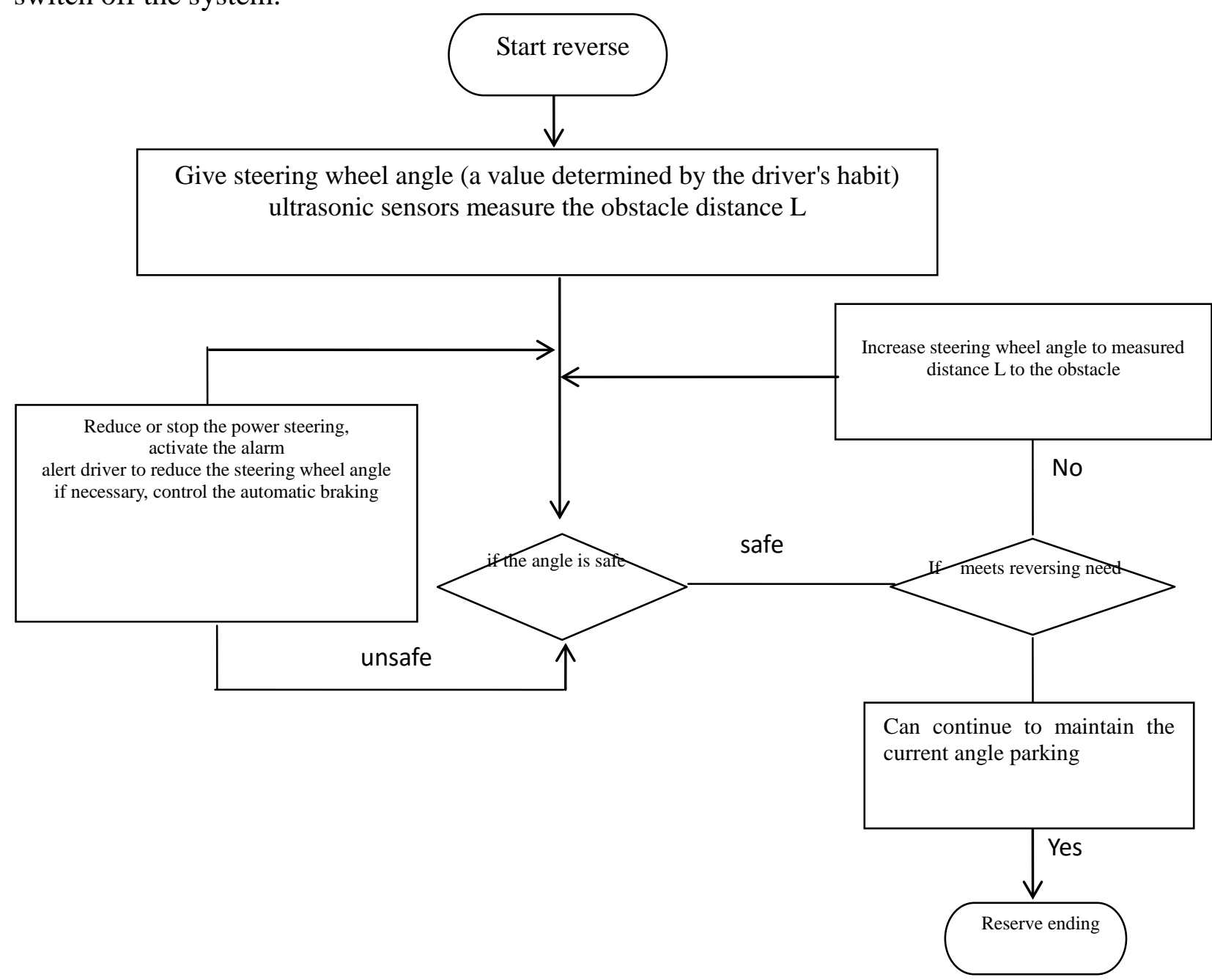

Figure 1 Principle diagram of car automatic reversing safety control System

Rear view mirror; rear wheel on behind

In the linear reverse mode, we mainly first adjust the angle of the rear view mirror, as shown in Figure. 3, the angle $\theta$ with is associated with the distance $\mathrm{x}$ from rear view mirror to the ground and the vertical distance $y$ from wheel on behind to the rear view mirror behind, as defined in formula $1-1$.

$$
\theta=\arctan \frac{y}{x}
$$

Under normal reverse mode, when the steering wheel when the angle is changed, according to the duration of the corner and the rate of change of angular change can be obtained, as shown in Formula 1-2[3].

$$
\begin{aligned}
& \frac{d \theta}{d t}=\frac{\Delta M}{I}=\frac{\left(M_{1}+M_{2}\right)-M_{0}}{I} \\
& M_{2}=f(n) * k, \mathrm{n}=g(b)
\end{aligned}
$$


By formula (1-3), hydraulic steering torque M2 can be calculated. $\theta \approx 10 \alpha, R=\sin \alpha^{*} L+B$

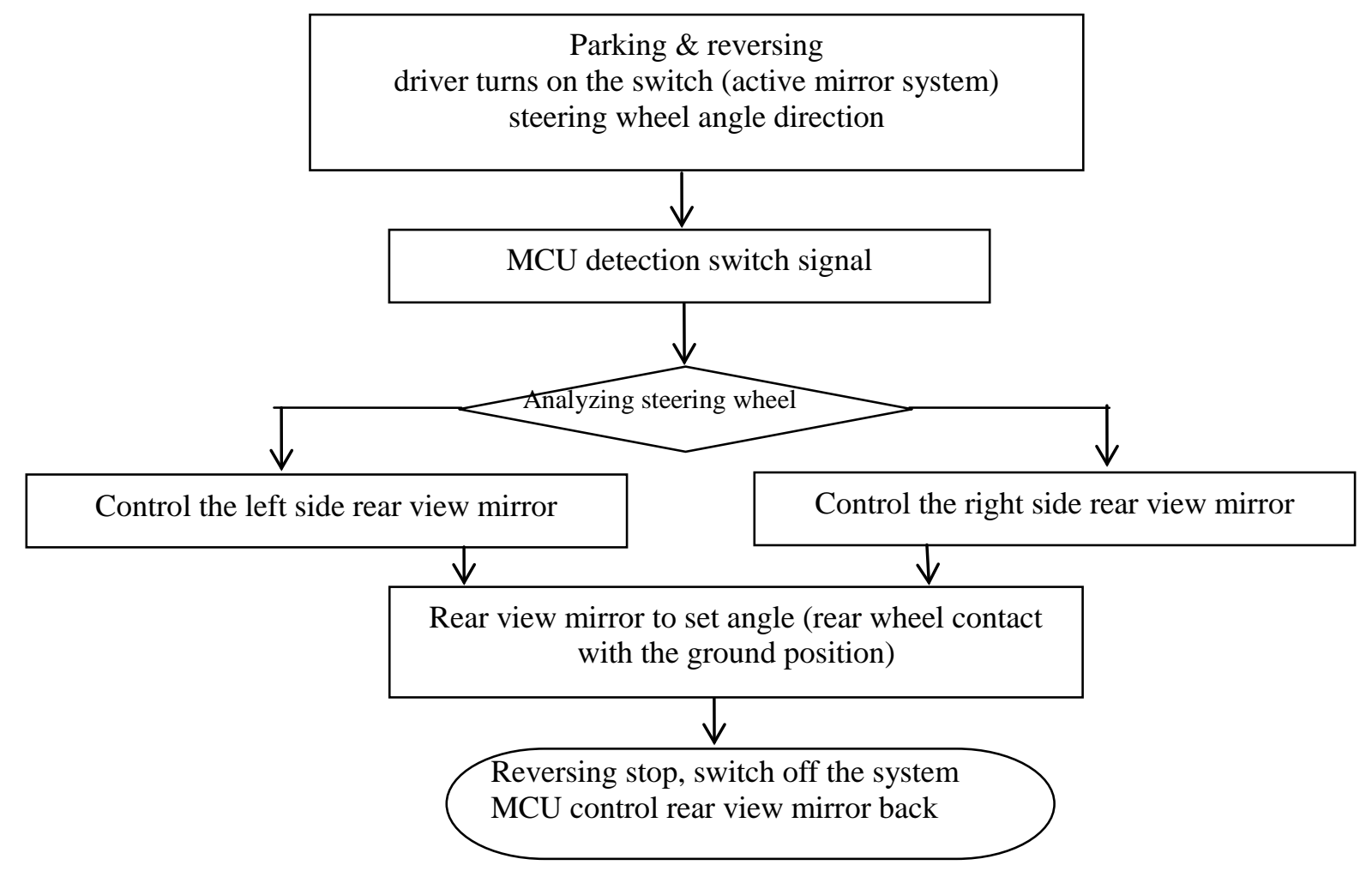

Figure 2. Auxiliary system working principle diagram in car reversing mode

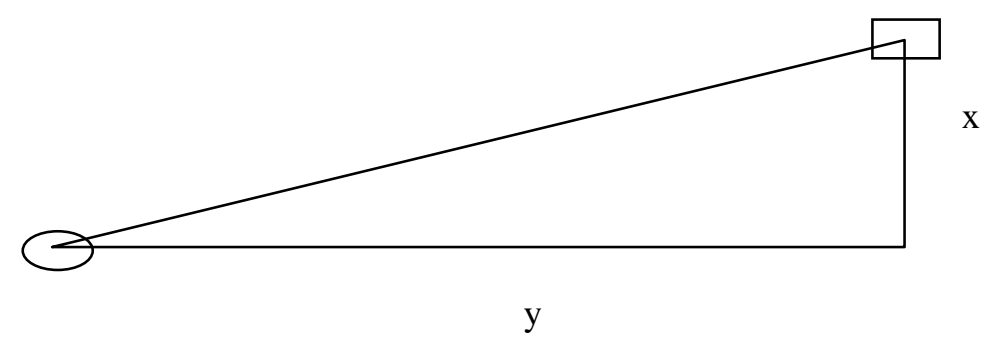

Figure3. Active rear view mirror data calculation

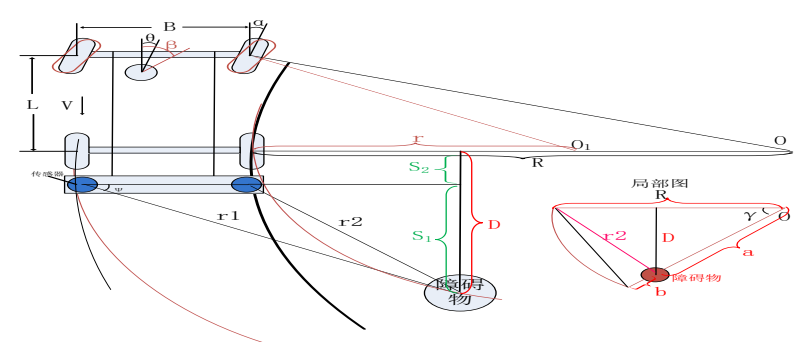

Figure 4. Active reverse geometry

If combine the above formula 1-2,1-3,1-4, that the relationship between the turning radius $\mathrm{R}$ and the angular rate of change can be figured out.

$$
\cos \psi=\frac{r_{1}^{2}+B^{2}-r_{2}^{2}}{2 r_{1} B}
$$




$$
S_{1}=\Gamma_{1}^{*} \sin \psi
$$

From formula 1-5, 1-6 , vertical distance between apparent obstacle and sensor of S1 can be known .

$$
D=S_{1}+S_{2}
$$

(S1 is the vertical distance between the obstacle and the sensor, S2 is the vertical distance between the rear wheel and sensor)

By the formula1-7 it can be figured out that $\mathrm{D}$ is vertical distance from apparent obstacle to the rear wheel[4].

$$
\begin{aligned}
\gamma & =\frac{V^{*} \Delta t}{R} \\
\mathrm{a} & =\frac{D}{\sin \gamma} \\
\mathrm{b} & =\mathrm{R}-\mathrm{a}
\end{aligned}
$$

When the above formula 1-7,1-8,1-9,1-10 simultaneous, a safe distance $\mathrm{b}$ from the turning radius $\mathrm{R}$ can be known.

When the car cornering at a constant speed, the steering wheel angle remains unchanged, the car with the radius of the circle will also be the same. Based on the rate of change of the steering wheel angle can be predicted turning radius $\mathrm{R}$, so that we can know the car trajectory, according to the ultrasonic sensor measures the distance, we can see that the obstacle is within the danger zone, according to the above mathematical model, we make $b>5 \mathrm{~cm}$ as safe distance[5].

\section{Components and implementation of System}

\section{Hardware Design}

a) Hardware circuit of system includes a sensor signal acquisition, alarm display section, and other electronically controlled hydraulic power section. Process of the system is probably shown in Figure 5.

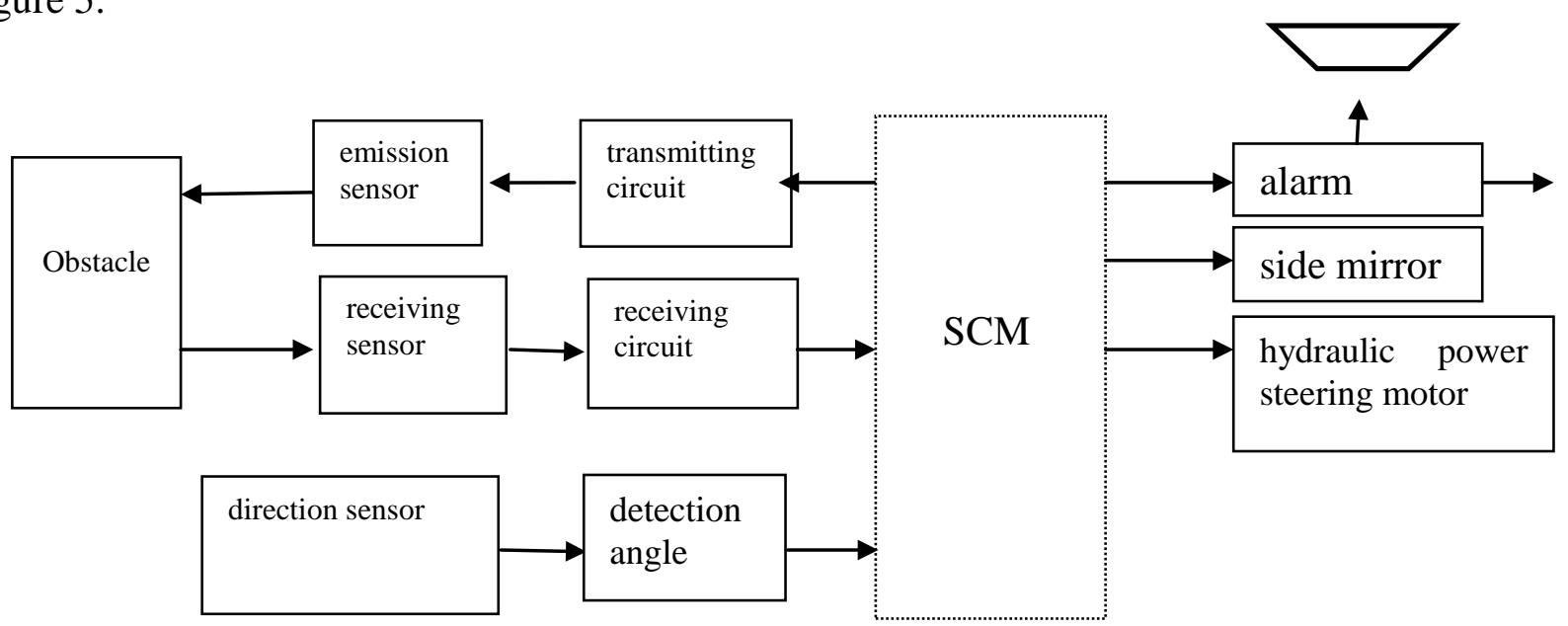

Figure 5. System hardware configuration diagram

b) Sensor signal acquisition

The system uses a serial trigger ranging mode of US-100 ultrasonic ranging module, which consists of an ultrasonic generator and an ultrasonic receiver. Ultrasonic generator ultrasonic oscillator and probe makes up of ultrasonic transmitter. an ultrasonic transmitter sonde by an external oscillator circuit ends generated by the ultrasonic oscillator, and after the microcontroller output frequency of the ultrasonic transducer needs to $40 \mathrm{KHz}$ square wave signal, the ultrasonic transmitter after launching the probe dual inverters treated Sasser, its waveform approximates a square wave; ultrasonic receiver after receiving the echo signal, the signal is amplified and transmitted to the microcontroller for processing principle shown in Figure 6 [6]. 


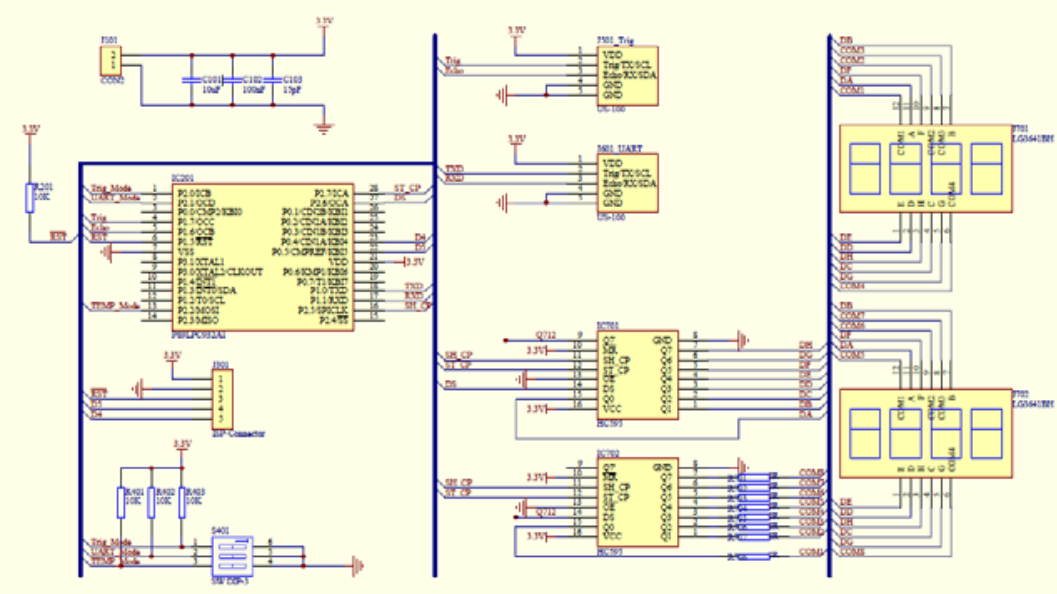

Figure 6 Ultrasonic sensor operating principle

A steering wheel angle sensor is an important part of the vehicle dynamics control system stability, accuracy and stability is directly related to traffic safety. It is mainly used to measure vehicle steering rotation angle of the steering wheel, the steering wheel angle sensor is a multi-turn absolute angle output mode, you can measure the absolute multi-turn angle of the steering wheel rotation. We measure the angle through the steering wheel angle sensor to the MCU and dealt with accordingly.

Vehicle speed sensor detects electric car speed, control computer with the input signal to make the appropriate judgment.

c) Warning display section

In the reference of car dashboard and following the vehicle under the premise of ergonomics, the system uses a combination of light and sound alarm mode. The visual and audible alarm improve driver safety state judge. Sound and light alarm system by LED and buzzer composition. Sound and light alarm device under the control of the ECU, ECU depending on the control signal transmitted, LED and beeper can be high and low frequency beeps and flashes to alert vehicle occupants, only when the car is in a safe state when, LED and buzzer will be turned off.

d) Electronically controlled hydraulic power section

Electronically controlled hydraulic power refers to replace the engine-driven hydraulic booster pump with electronic control system. Its working state is the ideal state calculated by the electronic control unit according to vehicle speed, steering angle signal. [7]The steering wheel angle changes with motor speed varies, when the steering track distance to the obstacle is less than the safe distance, adjust the motor speed, and then adjust the steering wheel angle. This paper uses a BLDC DC motor control module, control chip dsPIC33FJ16MC304, motor operating voltage of $24 \mathrm{~V}$, the master MCU module DAC output voltage value corresponding to the $\mathrm{AD}$ port motor control module to control the motor speed.

e) Automatically adjusts the rear view mirror section

Keep outputting a trigger level to change the angle of the rear view mirror according to the distance ultrasonic sensor detects.

\section{Software design of system}

According to the function car reversing active safety control system, part of the functions system software to achieve is as following: control transmission and reception of ultrasonic; ultrasonic round-trip time difference calculation, and then calculates the distance between the rear of the car and the obstacle; data processing, more cars the distance between the obstacle and the tail value and pre-set alarm threshold distance, the distance prompt and alarm. To find errors in the program easily, the system software is modular programming. The entire system software includes the main program, subprogram transmit receiving subroutine delay subroutine, ranging routines, display alarm subroutine, the subroutine active side mirror, steering wheel angle measurement subroutine, the steering system control subroutine modules, as Figure 7. 


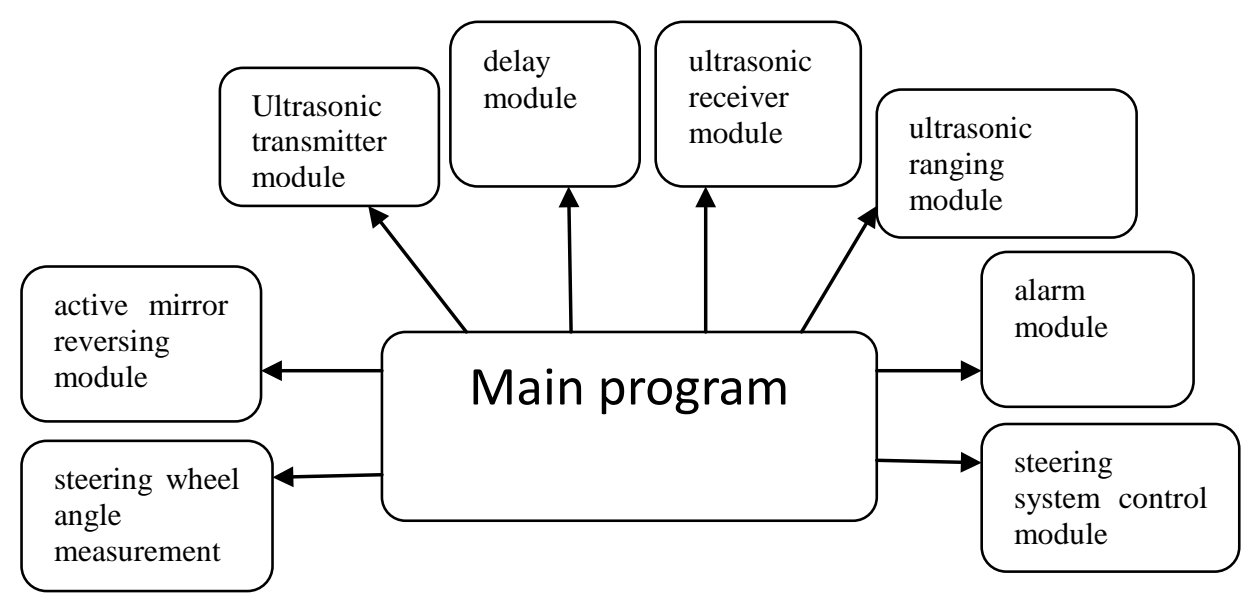

Figure 7. System software design

According to the system implementation process, the main program of software system configuration diagram is shown in Figure 8[8].

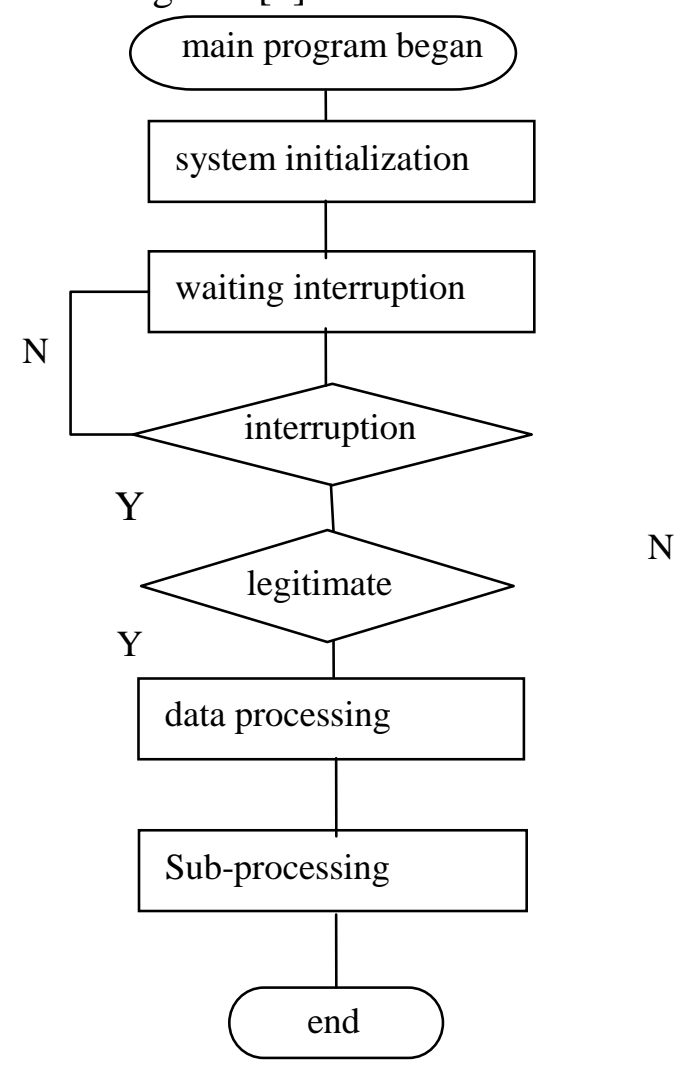

Figure8. The main program flowchart

\section{Results and demonstration}

The trial is the overall experiment on performance and functionality of each module unit consisting of automotive active safety reversing control system to verify that if can effectively measure the distance and observe the warning and the timely and effective realization of the steering ECU according to the results of the data control of the motor.

The results of the experimental tests are shown in Table 1.

(1) Results of the test is fully consistent with the actual situation and the expected alarm condition alarm, proving reversing automotive active safety control system logic alarm and control functions meet the requirements;

(2) After main control unit, ultrasonic ranging module working after several hours, although the chip fever, but in the normal range, does not affect the stability and reliability of system operation; 
(3) Serial communications and data transmission program run properly, proving the communication system design and software programming meet the design requirements;

(4) Sound and light alarm device is timely and eye-catching, ECU can realize the control of motor under dangerous situation[9].

In summary, the experimental verified that each module design of car reversing active safety control system is reasonable, reliable, running in good condition overall and in line with the design requirements.

Table 1 Results of experiment

\begin{tabular}{|c|c|c|c|c|c|c|c|c|}
\hline $\begin{array}{c}\text { Steering } \\
\text { case }\end{array}$ & Distance & $\begin{array}{c}\text { Expected } \\
\text { mirror }\end{array}$ & Actual mirror & Expected alarm & Actual alarm & $\begin{array}{c}\text { Expected } \\
\text { control }\end{array}$ & $\begin{array}{c}\text { Actual } \\
\text { control }\end{array}$ & $\begin{array}{c}\text { Work } \\
\text { state }\end{array}$ \\
\hline no & far & Adjust angle & Adjust angle & No alarm & No alarm & no & no & normal \\
\hline no & close & Adjust angle & Adjust angle & $\begin{array}{c}\text { Light\& } \\
\text { sound }\end{array}$ & Light\&sound & no & no & normal \\
\hline yes & far & No control & No control & No alarm & No alarm & no & no & normal \\
\hline yes & No close & No control & No control & $\begin{array}{c}\text { Light\& } \\
\text { sound }\end{array}$ & Light\&sound & no & no & normal \\
\hline yes & No far & No control & No control & Light\& sound & Light\&sound & ease & ease & normal \\
\hline yes & close & No control & No control & Light\& sound & Light\&sound & no & no & normal \\
\hline
\end{tabular}

\section{Summary}

This article combine chip technology, the active side mirror and distance sensor of high precision and high reliability, realize voice prompts through sound alarm circuit, use digital LED display the distance between the obstacle and information systems, and use hydraulically power steering control steering motor to change the active steering control cars. Active reversing safety control system is an effective means and tools of traffic management, which can improve the accuracy of vehicle detection distance, and conducive to the scientific management of transportation, reduce the driver's own quality requirements.

\section{References}

[1] Jishou Wen, etc. Progress in Intelligent Vehicle and abroad. China Transportation Research and Exploration in the third national conference of young scholars transportation, Wuhan, 2001.

[2] Liu Changli, Yang Guoqing, Xiang Zhongxiang. Japan's future security early warning system of an automobile, automotive electronics, 1999, 12 (l): 23.

[3] Matsumoto N, Tomizuka M. Vehicle Lateral Velocity and Yaw Rate Control with Two Independent Control Inputs [J] .Transactions of the ASME, 1992, 114 (12): 606-613.

[4] Hu Jiankai, Zhang Qianlin. Ultrasonic detection principle and method [J]. China University of Technology Press, 1993,10.

[5] Fan Shangchun. Sensor technology and its application [M]. Beijing: Beijing Aerospace University Press, 2010.

[6] Liu Guanghua, Buying Yong, Wang Jichan microcontroller-based precision ultrasonic ranging system [J] Instrument Technique and Sensor, 2007 (2): 66-68.

[7] Weng Lilang, Yang Li ultrasonic transducer driver and receiver circuits [J] Jimei University Natural Science Edition, 1998,3 (4): 60-64.

[8] Chenguang. Single-chip microcomputer principle and interface technology (Second Edition) [M] Wuhan: Huazhong University Press, Second Edition 1999.4: 22-29.

[9] Songxian, Miaojiong. Design and Implementation of embedded real-time operating system serial communication [J] Electronic Design Engineering, 2011,19 (1): 44. 